\section{Medidas de associação em \\ estudo transversal com \\ delineamento complexo: \\ razão de chances e razão de \\ prevalência}

\section{Association measures in cross- \\ sectional studies with complex \\ sampling: odds ratio and prevalence ratio}

Priscila Maria S. Bergamo Francisco'

Maria Rita Donalisio'

Marilisa Berti de Azevedo Barros ${ }^{1}$

Chester Luis Galvão Cesar ${ }^{2}$

Luana Carandina ${ }^{3}$

Moisés Goldbaum ${ }^{4}$

${ }^{1}$ Faculdade de Ciências Médicas - UNICAMP. Departamento de Medicina

Preventiva e Social.

${ }^{2}$ Faculdade de Saúde Pública - USP. Departamento de Epidemiologia.

${ }^{3}$ Faculdade de Medicina de Botucatu - UNESP. Departamento de Medicina em Saúde Pública.

${ }^{4}$ Faculdade de Medicina - USP. Departamento de Medicina Preventiva.

\section{Resumo}

O objetivo deste estudo foi apresentar e discutir a utilização das medidas de associação: razão de chances e razão de prevalências, em dados obtidos de estudo transversal realizado em 2001-2002, utilizando-se amostra estratificada por conglomerados em dois estágios $(\mathrm{n}=1.958)$. As razões de chances $\mathrm{e}$ razões de prevalências foram estimadas por meio de regressão logística não condicional e regressão de Poisson, respectivamente, utilizando-se o pacote estatístico Stata 7.0. Intervalos de confiança e efeitos do desenho foram considerados na avaliação da precisão das estimativas. Dois desfechos do estudo transversal com diferentes níveis de prevalência foram avaliados: vacinação contra influenza $(66,1 \%)$ e doença pulmonar referida $(6,9 \%)$. Na situação em que a prevalência foi alta, as estimativas das razões de prevalência foram mais conservadoras com intervalos de confiança menores. $\mathrm{Na}$ avaliação do desfecho de baixa prevalência, não se observaram grandes diferenças numéricas entre as estimações das razões de chances e razões de prevalência e errospadrão obtidos por uma ou outra técnica. $\mathrm{O}$ efeito do desenho maior que a unidade indicou que a amostragem complexa, em ambos os casos, aumentou da variância das estimativas. Cabe ao pesquisador a escolha da técnica e do estimador mais adequado ao seu objeto de estudo, permanecendo a escolha no âmbito epidemiológico.

Palavras-chave: Estudo transversal. Razão de chances. Razão de prevalências. 


\section{Abstract}

The objective for this paper was to present and discuss the use of odds ratios and prevalence ratios using real data with a complex sampling design. We carried out a cross-sectional study using data obtained from a two-stage stratified cluster sample from a study conducted in 2001-2002 ( $\mathrm{n}=$ 1,958). Odds ratios and prevalence ratios were obtained by unconditional logistic regression and Poisson regression, respectively, for later comparison using the Stata statistical package (v. 7.0). Confidence intervals and design effects were considered in the evaluation of the precision of estimates. Two outcomes of a cross-sectional study with different prevalences were evaluated: vaccination against influenza $(66.1 \%)$ and self-referred lung disease (6.9\%). In the high-prevalence scenario, using prevalence ratios the estimates were more conservative and we found narrower confidence intervals. In the low-prevalence scenario, we found no important numeric differences between the estimates and standard errors obtained using the two techniques. A design effect greater than one indicates that the sample design has increased the variance of the estimate. However, it is the researcher's task to choose which technique and measure to use for each data set, since this choice must remain within the scope of epidemiology.

Keywords: Cross-sectional studies. Odds ratio. Prevalence ratios.

\section{Introdução}

Estudos transversais com delineamento amostral complexo têm sido amplamente utilizados nas diferentes áreas do conhecimento. Particularmente na área da saúde pública, são crescentes os levantamentos que têm empregado essa técnica para coleta de dados ${ }^{1-2}$.

Desde 1960, pesquisas domiciliares com graus diferenciados de abrangência e complexidade já eram realizadas no país pelo Instituto Brasileiro de Geografia e Estatística (IBGE). Tais pesquisas utilizam amostras probabilísticas de domicílios com desenhos que incluem amostragem por conglomerados em dois estágios (setores censitários e domicílios) ou três estágios (municípios, setores censitários e domicílios), com estratificação das unidades primárias de amostragem (UPA's) ${ }^{3}$.

A amostragem por conglomerado é adotada, ao invés de a amostragem aleatória simples, principalmente por motivos de ordem prática e econômica ${ }^{4-5}$. No entanto, o sorteio da amostra por meio de conglomerados geralmente resulta em alteração na precisão das estimativas, que depende da composição interna dos conglomerados e da estratégia elaborada para o sorteio ${ }^{4,5}$. Nas análises realizadas com dados obtidos de tais delineamentos torna-se necessária a utilização de rotinas especiais de estimação e de testes de hipóteses que considerem o plano de amostragem ${ }^{5-6}$.

Softwares estatísticos convencionais em geral não consideram a estratificação, existência de conglomerados, probabilidades desiguais de seleção das unidades amostrais, ajustes para não-resposta e pósestratificação, características de delineamentos amostrais complexos ${ }^{4-7}$. Por ignorar tais aspectos, o uso desses softwares pode subestimar consideravelmente a variância associada a uma estimativa pontual, já que as estimativas pontuais dos parâmetros populacionais são impactadas pelo valor ponderado de cada observação ${ }^{7}$.

Procedimentos para análise de inquéritos populacionais de alguns programas es- 
tatísticos como o Stata, consideram o efeito do desenho amostral e permitem incorporar os pesos distintos das observações. ${ }^{2,4,5,8}$.

No que se refere à análise de dados, estudos epidemiológicos transversais com desfechos binários freqüentemente utilizam a regressão logística, cuja medida de associação é a razão de chances (odds ratio, $\mathrm{OR}$ ( $^{9-}$ ${ }^{12}$. No entanto, essa medida diferencia-se da razão de prevalências (RP), medida natural desses estudos ${ }^{10,12,13}$, especialmente quando os valores das prevalências são elevados.

A escolha entre as duas medidas na avaliação das associações em estudos transversais, bem como a técnica de análise para a obtenção das mesmas, vem sendo discutida na literatura epidemiológica ${ }^{9,10,12,14,15}$, já que elas podem ser discrepantes na avaliação de condições ou doenças comuns, freqüentemente focalizadas em estudos transversais ${ }^{11}$.

O objetivo deste estudo é apresentar e discutir a utilização da razão de chances e razão de prevalências, por meio de regressão logística e de Poisson, na análise de fatores associados à vacinação contra influenza (alta prevalência) e doença pulmonar (baixa prevalência) em uma amostra de idosos.

\section{Métodos}

Os modelos utilizados na análise dos dados pertencem à classe dos modelos lineares generalizados, indicados quando as variáveis estudadas não têm aderência à distribuição normal. Tais modelos são definidos por uma distribuição de probabilidade, membro da família exponencial de distribuições para a variável resposta, um conjunto de variáveis independentes descrevendo a estrutura linear do modelo e uma função de ligação entre a média da variável resposta e a estrutura linear. Em tais modelos estatísticos, a variável dependente é uma contagem e as variáveis independentes buscam explicar o comportamento da série ${ }^{16}$.

\section{Regressão Logística}

Existem situações em que se deseja modelar a probabilidade de um evento em função de um conjunto de variáveis explicativas qualitativas ou quantitativas. Na análise de regressão logística, a variável dependente (resposta) é uma variável aleatória dicotômica que assume o valor 1 se o evento de interesse ocorre ou 0 em caso contrário. $\mathrm{O}$ modelo utiliza a transformação logito (logaritmo neperiano do odds) para evitar que a função assuma valores negativos ${ }^{17}$ :

A medida (estimador) que expressa o risco é a razão de chances ou odds ratio ${ }^{9,11}$, a qual avalia a relação entre a chance de um indivíduo exposto possuir a condição de interesse, comparada à do não exposto. É um método bastante conhecido, disponível em diversos pacotes estatísticos e possui boas propriedades estatísticas ${ }^{9,10}$.

\section{Regressão de Poisson}

O modelo de Poisson desempenha importante papel na análise de dados em forma de contagens e é utilizado para analisar a associação de variáveis discretas e um conjunto de variáveis explicativas. Na epidemiologia, a regressão de Poisson é usada na análise de estudos longitudinais, onde o desfecho é uma contagem de episódios de um evento em um intervalo de tempo ${ }^{12}$. Na aplicação do modelo em estudos transversais, considera-se constante o tempo de seguimento. $\mathrm{O}$ uso da função de ligação "log" garante que os valores ajustados permaneçam no intervalo $[0, \infty)$.

A medida (estimador) que expressa o risco é a razão de prevalências, que mede a prevalência da condição de interesse no grupo de expostos em relação à prevalência no grupo dos não expostos ${ }^{13}$. O método tem sido aplicado em estudos epidemiológicos transversais ${ }^{12}$.

As diferentes técnicas foram analisadas em duas situações (prevalência $>50 \%$ e prevalência $<10 \%$ ) utilizando-se dados do "Inquérito de saúde de base populacional em municípios do estado de São Paulo" (ISA-SP), realizado no período de 2001 a $2002^{18}$. A pesquisa teve como base uma 
amostra probabilística da população, estratificada, por conglomerados e obtida em dois estágios de seleção. Para as análises realizadas no presente estudo foram utilizados dados da população de 60 anos e mais não institucionalizada, residente em área urbana, em domicílios particulares ou coletivos $(\mathrm{n}=1.958)$.

Estimou-se a associação entre vacinação contra influenza nos 12 meses que antecederam a pesquisa $(66,1 \%$ dos idosos referiram adesão à vacinação) ${ }^{19} \mathrm{e}$ variáveis sociodemográficas e de saúde, a saber: sexo, idade, escolaridade, tabagismo, auto-avaliação da saúde e relato de algumas doenças crônicas. Também foi verificada a associação entre doença pulmonar referida (prevalência de $6,9 \%)^{20}$ e as mesmas variáveis independentes selecionadas para vacinação.

Considerando-se que a variável idade está fortemente associada à prevalência da vacinação e de doenças crônicas, todas as prevalências foram ajustadas por idade antes de se proceder às comparações. A análise múltipla foi baseada em razões de chances (OR), utilizando-se a regressão logística não condicional e razões de prevalências (RP) e intervalos de confiança robustos, pela regressão de Poisson ${ }^{12}$. No modelo múltiplo foram consideradas as variáveis que tiveram um valor de $\mathrm{p}<0,20$ na análise univariada, realizada por meio do teste $\chi^{2}$ e a variável permaneceu no modelo se $\mathrm{p}<$ 0,05 . Na análise dos dados foram utilizados procedimentos do programa Stata (versão 7.0) para inquéritos populacionais ${ }^{8}$.

O projeto deste estudo foi aprovado pelo Comitê de Ética em pesquisa da Faculdade de Ciências Médicas da UNICAMP sob o parecer no $369 / 2000$.

\section{Resultados}

No que se refere à avaliação dos fatores associados à vacinação contra influenza, situação em que a prevalência é alta, ainda que as variáveis idade, escolaridade, hipertensão arterial e diabetes tenham apresentado significância estatística, independente da medida de associação utilizada, observa-se que para todas elas a razão de prevalências apresentou estimativas pontuais mais conservadoras e intervalos mais compactos em relação à razão de chances. Nessa situação, esse estimador pode estar superestimando a magnitude da associação (Tabela 1).

Como pode ser observado na Tabela 2, quando a prevalência da doença é baixa, as estimativas pontuais e por intervalo são bem próximas, situação em que ambas as medidas representam bem a magnitude da associação e as diferenças residem apenas na sua interpretação.

Na Tabela 3 observam-se os resultados da análise múltipla da situação onde a prevalência da condição estudada é alta. O modelo final, obtido pelas diferentes técnicas, foi o mesmo. Mediante regressão logística, estiveram independentemente associados à vacinação: idade igual ou superior a 70 anos, escolaridade inferior a 9 anos de estudo e hipertensão arterial referida. A partir da regressão de Poisson, idade e hipertensão apresentaram-se estatisticamente associadas à vacinação, e a escolaridade esteve no limite da significância estatística.

Na situação de baixa prevalência, ambas as técnicas também apresentaram resultados semelhantes, ou seja, apenas o tabagismo e a pior avaliação da saúde estiveram independentemente associados à doença pulmonar referida (Tabela 4). Quanto à interpretação das medidas, com relação à razão de chances, os fumantes ou ex-fumantes e aqueles com pior avaliação da saúde têm chance superior de apresentar doença pulmonar do que os não fumantes e com boa avaliação da própria saúde, respectivamente. Considerando a razão de prevalências como medida de associação, pode-se dizer que a prevalência de doença pulmonar é maior entre os idosos fumantes ou ex-fumantes e naqueles com pior avaliação da saúde quando comparada aos não fumantes e com boa avaliação da saúde.

No que se refere à magnitude das medidas, em ambas as situações analisadas as razões de prevalências apresentaram estimativas mais conservadoras e intervalos mais compactos em relação à razão de chan- 
Tabela 1 - Prevalência (P), razão de chances (OR) e razão de prevalências (RP) de vacinação contra influenza segundo características sociodemográficas e de saúde. ISA-SP, 2001-2002.

Table 1 - Prevalence (P), odds ratio (OR) and prevalence ratio (RP) for influenza vaccination according to demographic, social and health-related characteristics. ISA-SP, 2001-2002.

\begin{tabular}{|c|c|c|c|c|c|}
\hline Variáveis e Categorias & $\mathrm{N}$ & $\mathrm{P}(\%)$ & $\mathrm{p}^{*}$ & OR (IC 95\%) & RP (IC 95\%) \\
\hline \multicolumn{6}{|l|}{ Sexo } \\
\hline Masculino & 905 & 67,1 & & 1 & 1 \\
\hline Feminino & 1.003 & 65,4 & 0,52 & $0,90(0,71-1,14)$ & $0,96(0,89-1,05)$ \\
\hline \multicolumn{6}{|l|}{ Faixa etária } \\
\hline 60 a 69 & 1.061 & 62,0 & & 1 & 1 \\
\hline 70 e mais & 847 & 71,3 & 0,01 & $1,52(1,13-2,05)$ & $1,15(1,04-1,27)$ \\
\hline \multicolumn{6}{|l|}{ Escolaridade } \\
\hline Nove ou mais anos de estudo & 334 & 56,3 & & 1 & 1 \\
\hline Até 9 anos de estudo & 1.566 & 68,5 & 0,02 & $1,66(1,07-2,57)$ & $1,21(1,01-1,45)$ \\
\hline \multicolumn{6}{|l|}{ Tabagismo } \\
\hline Não fumante & 1.015 & 67,4 & & 1 & 1 \\
\hline Fumante ou ex-fumante & 889 & 64,5 & 0,30 & $0,90(0,71-1,14)$ & $0,96(0,89-1,05)$ \\
\hline \multicolumn{6}{|l|}{ Auto-avaliação da saúde } \\
\hline Excelente/Muito boa/Boa & 1.595 & 66,6 & & 1 & 1 \\
\hline Ruim/Muito ruim & 268 & 64,6 & 0,62 & $0,86(0,60-1,23)$ & $0,95(0,84-1,08)$ \\
\hline \multicolumn{6}{|c|}{ Relato das seguintes doenças/condições crônicas } \\
\hline Hipertensão & 917 & 70,5 & 0,01 & $1,46(1,07-1,99)$ & $1,14(1,02-1,26)$ \\
\hline Diabetes & 286 & 73,9 & 0,04 & $1,55(1,01-2,36)$ & $1,14(1,02-1,28)$ \\
\hline Artrite/reumatismo/artrose & 488 & 70,9 & 0,08 & $1,33(0,93-1,88)$ & $1,09(0,98-1,22)$ \\
\hline Doença do coração & 293 & 67,4 & 0,70 & $1,00(0,70-1,42)$ & $1,00(0,89-1,12)$ \\
\hline Doença pulmonar & 139 & 67,9 & 0,71 & $1,08(0,67-1,74)$ & $1,03(0,88-1,20)$ \\
\hline
\end{tabular}

ces. Quanto ao efeito do desenho, a variável escolaridade associada à vacinação apresentou importante grau de homogeneidade intra-conglomerado Deff=3,19 e Deff=3,26, respectivamente (Tabela 3). De modo geral, o efeito do desenho foi maior que a unidade. Neste estudo, independente da técnica de análise utilizada, o plano de amostragem por conglomerados teve impacto sobre a variância das estimativas?

\section{Discussão}

Considerando-se que a medida de associação em estudos transversais é a razão de chances ou a razão de prevalências, o presente trabalho utilizou duas técnicas de modelagem na análise epidemiológica de dados reais com prevalência menor que $10 \%$ e superior a $50 \%$ para obter e comparar tais indicadores.

Alternativas para análise de dados transversais têm sido propostas e utilizadas por vários autores em diversos países ${ }^{9,13,21} \mathrm{e}$, mais recentemente no Brasil, destacandose a contribuição de Barros e Hirakata ${ }^{12}$, que discutem estratégias apropriadas para produzir estimativas pontuais e por intervalos para as razões de prevalências. Particularmente as razões de prevalências e 
Tabela 2 - Prevalência (P), razão de chances (OR) e razão de prevalências (RP) para doença pulmonar segundo características sociodemográficas e de saúde. ISA-SP, 2001-2002.

Table 2. Prevalence (P), odds ratio (OR) and prevalence ratio (RP) for pulmonary disease according to demographic, social and health-related characteristics. ISA-SP, 2001-2002.

\begin{tabular}{|c|c|c|c|c|c|}
\hline Variáveis e Categorias & $\mathrm{N}$ & $\mathrm{P}(\%)$ & $\mathrm{p}^{*}$ & OR (IC 95\%) & RP (IC 95\%) \\
\hline \multicolumn{6}{|l|}{ Sexo } \\
\hline Masculino & 929 & 7,98 & & 1 & 1 \\
\hline Feminino & 1.028 & 6,18 & 0,22 & $0,75(0,48-1,18)$ & $0,77(0,50-1,17)$ \\
\hline \multicolumn{6}{|l|}{ Faixa etária } \\
\hline 60 a 69 & 1.092 & 6,66 & & 1 & 1 \\
\hline 70 e mais & 865 & 7,32 & 0,67 & $1,11(0,69-1,78)$ & $1,10(0,71-1,71)$ \\
\hline \multicolumn{6}{|l|}{ Escolaridade } \\
\hline Nove ou mais anos de estudo & 347 & 5,82 & & 1 & 1 \\
\hline Até 9 anos de estudo & 1.602 & 6,91 & 0,54 & $1,24(0,61-2,54)$ & $1,23(0,63-2,40)$ \\
\hline \multicolumn{6}{|l|}{ Tabagismo } \\
\hline Não fumante & 1.044 & 4,74 & & 1 & 1 \\
\hline Fumante ou ex-fumante & 909 & 9,72 & $<0,01$ & $2,19(1,40-3,42)$ & $2,07(1,36-3,15)$ \\
\hline \multicolumn{6}{|l|}{ Auto-avaliação da saúde } \\
\hline Excelente/Muito boa/Boa & 1.632 & 5,77 & & 1 & 1 \\
\hline Ruim/Muito ruim & 275 & 14,58 & $<0,01$ & $2,80(1,66-4,73)$ & $2,54(1,60-4,04)$ \\
\hline \multicolumn{6}{|l|}{ Vacinação contra influenza } \\
\hline Não & 680 & 6,62 & & 1 & 1 \\
\hline Sim & 1.227 & 7,20 & 0,71 & $1,08(0,68-1,74)$ & $1,08(0,69-1,68)$ \\
\hline \multicolumn{6}{|c|}{ Relato das seguintes doenças/condições crônicas² } \\
\hline Hipertensão & 940 & 7,17 & 0,78 & $1,07(0,64-1,80)$ & $1,07(0,66-1,73)$ \\
\hline Diabetes & 292 & 6,95 & 0,12 & $1,01(0,54-1,86)$ & $1,01(0,57-1,78)$ \\
\hline Artrite/reumatismo/artrose & 505 & 8,62 & 0,17 & $1,38(0,86-2,23)$ & $1,35(0,87-2,10)$ \\
\hline Doença do coração & 297 & 10,10 & 0,12 & $1,64(0,84-3,20)$ & $1,57(0,86-2,88)$ \\
\hline
\end{tabular}

intervalos de confiança robustos por meio de regressão de Poisson vêm sendo utilizadas em análises de estudos transversais complexos $^{22}$. A ampliação da utilização de diferentes técnicas de análise para dados transversais com desfechos binários permite aos pesquisadores que a escolha da medida apropriada seja de fato, baseada em conceitos epidemiológicos.

Nesse estudo, as estimativas pontuais para as associações significativas nas análises simples e múltipla mostraram regularidade de superestimação da razão de chances em comparação com as razões de prevalências em ambas as situações. Com relação aos intervalos de confiança estimados, tanto para as medidas brutas (dados não apresentados) quanto para as ajustadas, aqueles correspondentes às razões de chances sempre foram mais amplos, ou seja, menos precisos.

$\mathrm{Na}$ análise múltipla, considerando-se o desfecho de baixa prevalência, houve pouca diferença entre a razão de chances e a razão de prevalências, conforme observado por outros autores que compararam tais medi- 
Tabela 3 - Modelos de regressão múltipla para vacinação contra influenza em idosos. ISA-SP, 2001-2002.

Table 3 - Multivariate regression models for influenza vaccination among the elderly. ISA-SP, 2001-2002.

\begin{tabular}{|c|c|c|c|c|c|}
\hline Modelos & Medida & Erro-padrão & Valores $\mathrm{p}$ & IC 95\% & Deff \\
\hline Regressão logística & OR & & & & \\
\hline Idade igual ou maior que 70 anos & 1,47 & 0,22 & 0,01 & $1,09-1,99$ & 2,26 \\
\hline Escolaridade igual ou inferior a 9 anos & 1,57 & 0,34 & 0,04 & $1,02-2,42$ & 3,19 \\
\hline Hipertensão arterial referida & 1,39 & 0,21 & 0,03 & $1,03-1,87$ & 2,31 \\
\hline Regressão de Poisson & $\mathrm{RP}$ & & & & \\
\hline Idade igual ou maior que 70 anos & 1,13 & 0,05 & 0,01 & $1,03-1,25$ & 2,19 \\
\hline Escolaridade igual ou inferior a 9 anos & 1,18 & 0,11 & 0,06 & $1,00-1,41$ & 3,26 \\
\hline Hipertensão arterial referida & 1,11 & 0,05 & 0,03 & $1,01-1,23$ & 2,28 \\
\hline
\end{tabular}

Tabela 4 - Modelos de regressão múltipla para doença pulmonar em idosos. ISA-SP, 2001-2002.

Table 4 - Multivariate regression models for pulmonary disease among the elderly. ISA-SP, 2001-2002.

\begin{tabular}{lccccc}
\hline Modelos & Medida & Erro-padrão & Valores $\mathrm{p}$ & IC 95\% & Deff \\
\hline Regressão logística & $\mathrm{OR}$ & & & & \\
Fumante ou ex-fumante & 2,03 & 0,44 & 0,01 & $1,32-3,12$ & 1,36 \\
Auto-avaliação da saúde ruim ou muito ruim & 2,75 & 0,71 & $<0,01$ & $1,65-4,58$ & 1,51 \\
$\begin{array}{l}\text { Regressão de Poisson } \\
\text { Fumante ou ex-fumante }\end{array}$ & $\mathrm{RP}$ & & & & \\
$\begin{array}{l}\text { Auto-avaliação da saúde como ruim ou } \\
\text { muito ruim }\end{array}$ & 1,92 & 0,38 & 0,01 & $1,29-2,84$ & 1,36 \\
\hline
\end{tabular}

OR (IC 95\%) = Razão de chances (Intervalo de confiança em nível de 95\%)/odds ratio (95\% confidence interval)

RP (IC 95\%) = Razão de prevalência (Intervalo de confiança em nível de 95\%)/prevalence ratio (95\% confidence interval).

Deff: Efeito do desenho/design effect

das $^{10}$. Para ambos os modelos, o tabagismo atual ou anterior e a pior avaliação da própria saúde estiveram independentemente associados à doença pulmonar referida. Entretanto, muitos estudos transversais referem-se a desfechos com alta prevalência ${ }^{11}$ e no caso particular da vacinação contra influenza, analisada neste estudo, pode-se observar menor precisão da razão de chances, apesar dos modelos finais serem os mesmos mediante as diferentes técnicas de análise.

Vale lembrar a importância de se considerar o conglomerado na análise de dados oriundos de desenhos complexos, já que estimativas provenientes de uma amostra aleatória simples produziriam resultados subestimados para os erros-padrão e respectivos intervalos de confiança ${ }^{5,7}$. Neste estudo, o plano de amostragem por conglomerados teve impacto sobre a variância das estimativas, indicando que mais informações seriam necessárias para garantir a mesma precisão que se obteria por meio de um sorteio por amostragem aleatória simples com reposição ${ }^{5}$.

Do ponto de vista estatístico é adequada a utilização das razões de prevalências e intervalos de confiança robustos por meio de regressão de Poisson em estudos epidemiológicos transversais com delineamentos complexos envolvendo variáveis categóricas, independentemente da prevalência da condição estudada. Deve-se considerar, 
no entanto, que delineamentos transversais atendem a diversos propósitos e as aplicações podem sugerir o uso de diferentes medidas em diferentes contextos ${ }^{10}$.

No caso da vacinação, cujo propósito é descrever a prevalência segundo subgrupos específicos, a utilização da razão de prevalências parece mais apropriada, com maior capacidade explicativa do que a razão de chances, embora as duas medidas forneçam respostas na mesma direção ${ }^{13}$. Particularmente em aplicações em que se deseja trabalhar com desfechos que não necessariamente "doença", o uso da razão de prevalências como descritor do estado é uma medida mais "natural" e inteligível ${ }^{10}$.

Por outro lado, a doença pulmonar é um evento de baixa prevalência e, nesse caso, a razão de chances é numericamente similar à razão de prevalências não havendo diferença entre a utilização de uma ou outra medida $^{9,10,15}$. Além disso, dados de estudos transversais podem ser usados para estimar a razão de densidade de incidência, já que sob algumas suposições (desfecho raro em todos os níveis de exposição em uma coorte fechada ou tempo de duração da doença igual no grupo de expostos e não expostos), a razão de chances é um estimador da razão de densidade de incidência ${ }^{10,22,23}$.

A discrepância entre as medidas depende fortemente de ambos, prevalência da doença/condição estudada e prevalência da exposição/fatores, sendo a prevalência da doença/condição mais importante do ponto de vista quantitativo ${ }^{10}$. Portanto, deve-se considerar que este estudo se deu em circunstâncias especiais, analisando-se situações com prevalências específicas, na ausência de interação e com apenas uma variável de confusão (idade), tornando-se necessário verificar a adequação do uso da técnica em outros contextos.

No que se refere à interpretação das medidas, é importante lembrar que no contexto de estudos transversais, a razão de chances e a razão de prevalências não podem ser apresentadas como uma mesma medida ${ }^{15}$. A razão de chances expressa quantas vezes é maior a chance de encontrar a condição estudada entre os expostos em relação aos não expostos. A razão de prevalências estima uma probabilidade relativa de aleatoriamente selecionar um indivíduo e ele apresentar a condição estudada no período ${ }^{21}$.

Quanto à magnitude, na comparação de dados de diferentes estudos deve-se estar atento à medida de associação utilizada considerando a possibilidade de superestimativa do odds que se move em outra escala de medida e não compara proporções, mas chances. A relação matemática entre a razão de chances e razão de prevalências é uma curva quadrática ${ }^{10}$.

\section{Conclusões}

As medidas de associação avaliadas neste estudo têm sua função específica em estudos transversais e sua escolha deve permanecer no âmbito epidemioló$\operatorname{gico}^{10,15,23,24}$.

Particularmente no contexto da Saúde Pública, tanto a interpretação quanto a magnitude das medidas devem ser consideradas, na medida em que eventuais distorções podem interferir no planejamento de ações e alocação de recursos.

O estudo mostrou que, conforme o delineamento da pesquisa, a natureza e a freqüência do evento e das exposições estudadas, ambas as medidas de associação podem ser utilizadas, considerando sua capacidade explicativa e seus limites no contexto epidemiológico da investigação. Cabe ao pesquisador escolher a técnica $\mathrm{e}$ a medida mais adequada ao seu objeto de investigação e ser coerente na utilização e interpretação da medida escolhida. 


\section{Referências}

1. Cordeiro R. Efeito do desenho em amostragem de conglomerado para estimar a distribuição de ocupações entre trabalhadores. Rev Saúde Pública 2001; 35: 10-5.

2. Sousa MH, Silva NN. Estimativas obtidas de um levantamento complexo. Rev Saúde Pública 2003; 37: 622-70.

3. Bianchini ZM, Albieri S. Uma revisão dos principais aspectos dos planos amostrais das pesquisas domiciliares realizadas pelo IBGE. Revista Brasileira de Estatística 1999; 60: 7-23.

4. Korn EL, Graubard BI. Epidemiologic studies utilizing surveys: accounting for the sampling design. Am J Public Health 1991; 81: 1166-73.

5. Carlson BL. Software for statistical analysis of sample survey data. In: Armitage P, Colton T, editors. Encyclopedia of biostatistics, [serial on line] 1998. Disponível em < http://www.fas.harvard.edu/ stats / survey-soft/blc_eob.html>. Acessado em 03 de novembro de 2004 .

6. Pessoa DGC, Silva PLN, Duarte RPN. Análise estatística de dados de pesquisas por amostragem: problemas no uso de pacotes-padrão. Revista Brasileira de Estatística 1997; 58: 53-75.

7. Brogan DJ. Pitfalls of using standard statistical software packages for sample survey data. In: Armitage P, Colton T, editors. Encyclopedia of biostatistics, [serial on line] 1998. Disponível em <http://www.fas.harvard. edu/ stats/survey-soft/blc_eob.html>. Acessado em 08 de agosto de 2005.

8. StataCorp 2003. Stata Statistical Software: Release 8.0 College Station. TX: Stata Corporation.

9. Lee J. Odds ratio or relative risk for cross-sectional data? Int J Epidemiol 1994; 23: 201-3.

10. Zocchetti C, Consonni D, Bertazzi P. Relationship between prevalence rate ratios and odds ratios in crosssectional studies. Int J Epidemiol 1997; 26: 220-3.

11. Thompson ML, Myers JE, Kriebel D. Prevalence odds ratio or prevalence ratio in the analysis of cross sectional data: what is to be done? Occup Environ Med 1998; 55: 272-7.

12. Barros AJ, Hirakata VN. Alternatives for logistic regression in cross-sectional studies: an empirical comparison of models that directly estimate the prevalence ratio. BMC Med Res Methodol 2003; 3: 21.
13. Schiaffino A, Rodriguez M, Pasarin MI, Regidor E, Borrell C, Fernandez E. Odds ratio or prevalence ratio? Their use in cross-sectional studies. Gac Sanit 2003; 17: 70-4.

14. Stromberg U. Prevalence odds ratio or prevalence ratio. Occup Environ Med 1994; 51: 143-4.

15. Osborn J, Cattaruzza MS. Odds ratio and relative risk for cross-sectional data. Int J Epidemiol 1995; 24: 464-5.

16. Conceição GMS, Saldiva PHN, Singer JM. Modelos GLM e GAM para análise da associação entre poluição atmosférica e marcadores de morbi-mortalidade: uma introdução baseada em dados da cidade de São Paulo. Rev Bras Epidemiol, 2001; 4: 206-19).

17. Hosmer DW, Lemeshow S. Applied logistic regression. New York: John Wiley \& Sons; 1989.

18. Cesar CLG; Carandina L; Alves MCGP; Barros MBA; Goldbaum M. Saúde e condição de vida em São Paulo Inquérito multicêntrico de saúde no Estado de São Paulo - ISA-SP. Faculdade de Saúde Pública USP; 2005.

19. Francisco PMSB, Donalisio MR, Barros MBA, César CLG, Carandina L, Goldbaum M. Fatores associados à vacinação contra influenza em idosos. Rev Panam Salud Publica 2006; 19: 259-64.

20. Francisco PMSB, Donalisio MR, Barros MBA, César CLG, Carandina L, Goldbaum M. Fatores associados à doença pulmonar em idosos. Rev Saúde Publica 2006; 40: 428435.

21. Mendoza-Sassi R, Béria JU, Barros AJD. Outpatient health service utilization and associated factors: a population-based study. Rev Saúde Pública 2003; 37: 372-8.

22. Greenland S. Interpretation and choice of effect measures in epidemiologic analyses. Am J Epidemiol 1987; 125: 761-8.

23. Pearce N. Effect measures in prevalence studies. Environ Health Perspective 2004; 112: 1047-50.

Recebido em: 04/07/07 Versão final reapresentada em: 11/03/08 Aprovado em: 06/05/08 